\title{
A Case Study of Medical Data Classification Using Hybrid Adboost KNN along with Krill Herd Algorithm (KHA)
}

\author{
Dudekula Mahammad Rafi ${ }^{1 *}$, Chettiar Ramachandra Bharathi ${ }^{2}$ \\ ${ }^{1}$ Vel Tech Rangarajan Dr. Sagunthala R\&D Institute of Science and Technology, Avadi, Chennai, Tamil; Vivekananda \\ Institute of Engineering \& Technology, JNTU University, Hyderabad, India \\ ${ }^{2}$ Department of ECE, Vel Tech Rangarajan Dr. Sagunthala R\&D Institute of Science and Technology, Avadi, Chennai, Tamil \\ Nadu, India
}

Corresponding Author Email: rafiveltech@gmail.com

https://doi.org/10.18280/isi.240111

Received: 22 November 2018

Accepted: 16 January 2019

\section{Keywords:}

case study investigation, medical classification, krill herd algorithm, hybrid Adaboost k-nearest neighbor, accuracy, sensitivity and specificity

\begin{abstract}
This paper studies Medical classification challenging process methods using data ming, Hybrid Adboost KNN along with Krill Herd Algorithm (KHA) is applied to breast cancer medical data mining. Many death cases are happened due to breast cancer among ladies around the world, here tumor can identify as caused by disease. The data we study is collected from patients with breast cancer disease from hospitals. It is an ambiguous optimization problem and which is provide diagnosis aid effectiveness. It has 198 records of 34 attributes each. We use Krill Herd Algorithm to optimal features selection and Hybrid Adboost KNN is used to Classification. Case Study investigated the data mining methods and determine the Breast Cancer illness. The case study performance is evaluated in terms of accuracy, sensitivity and specificity. The Case Study will be implemented in Python software.
\end{abstract}

\section{INTRODUCTION}

The target of information mining is to distinguish substantial, novel, possibly valuable, and justifiable relationships and examples in existing information. Finding valuable examples in information is known by names (e.g., Knowledge extraction, data revelation, data reaping, and information design preparing). The expression "information mining" is basically utilized by analysts, database specialists, and the business networks. The term Knowledge Discovery in Databases (KDD) alludes to the general procedure of finding valuable information from information. Information mining is data extraction from database. Information mining systems are utilized to get right restorative analysis.

Arrangement of information mining frameworks should be possible as indicated by the sort of information sources mined, database included, the sort of learning found, and mining strategies utilized. Order is the association of information in given classes. Arrangement approaches ordinarily

utilize a preparation set where every one of the articles are as of now connected with realized class marks. The grouping calculation gains from the preparation set and manufactures a model. The model is utilized to characterize new items.

When a Classification show is constructed dependent on a preparation set, the class mark of an article can be predicted dependent on the trait estimations of the item and property estimations of the classes. Grouping is like Classification, bunching is the association of information in classes. In any case, the test increments as the enthusiasm for information mining develops quickly. As information digging systems for therapeutic information grouping has not been completely examined, there is an incredible potential for further work and intriguing bearings for research.

Krill Nearest Neighbor (KNN) strategy has been utilized in applications, for example, information mining, measurable example acknowledgment, picture preparing, acknowledgment of penmanship, Electrocardiography (ECG) infection order. The Krill Nearest Neighbor (KNN) strategy is an occurrence based learning technique that stores every single accessible datum set and orders new informational index dependent on similitude measure.

Half and half Adaboost Krill Nearest Neighbor (KNN) is a classifier. The Hybrid Adaboost Krill Nearest Neighbor $(\mathrm{KNN})$ arrangement limiting the preparation set blunder and boosting the edge so as to accomplish the best speculation capacity. Because of its profitable nature, Hybrid Adaboost Krill Nearest Neighbor (KNN) has been connected to a wide scope of order errands. Specifically, Hybrid Adaboost Krill Nearest Neighbor (KNN) has been to perform great on numerous restorative analysis undertakings. Nonetheless, there is as yet a requirement for improving the Hybrid Adaboost Krill Nearest Neighbor (KNN) classifier's execution. Anyway the exchange of basically sick patients requires great coordination to give the symptomatic apparatuses and the most suitable treatment for their conditions [17]. Hence it is very important to classify the disease on time and thereby providing treatments to the patients for risk avoidance. Sometimes same properties with the same symptoms of diseases required different treatments. Therefore accurate decisions and classification of data is required.

Medical data are mounting at hospitals, health centers and clinics. Eventually the quantity of information in the medical domain is increasing in recent decades. Restorative information contain data about research center test outcomes, tolerant socioeconomics, drug store data, radiology reports and pictures, pathology reports, emergency clinic affirmation, release and exchange dates, release rundowns and 
advancement notes. An important concern of the healthcare industry is to provide individualized patient care and not to collect or maintain data. However medical data is necessary for decision making, drug administration, diagnosis, statistical analysis and evidence. Hence efficient and effective techniques are required to extract useful information from this data.

The goal of data classification is to assign input data to one of a finite number of classes. The process of designing a data classifier design starts with data collection for any real world problem and then it undergoes a series of steps as given in figure 1 .

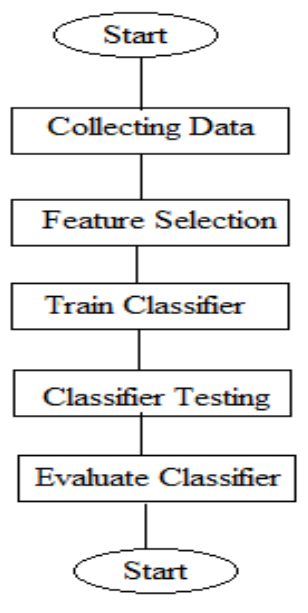

Figure 1. Data classifier design

An information classifier initially requires choice of features for every issue space. Great order execution requires choice of successful features and determination of a classifier that can make restricted preparing information, memory and figuring power.

Researchers attempted to use contrasting methods to hint at progress accuracy of data request. It is central to perform danger data course of action with a particular ultimate objective to perceive the disease. In this way to crush those issues our proposed procedure is used. Here at first the predealing will be associated with expel accommodating data and to change over sensible model from rough helpful datasets. In the wake of preprocessing for perfect assurance of features ACO based SVM is used. This system portrays the tumor data as run of the mill and sporadic. From this time forward our proposed system decisively arranges the tumor data using perfect features.

From this time forward to crush the issue a couple of new methods make sense of how to dissect infection in a totally data driven way, using multivariate course of action or backslide to clearly layout imaging data to end. These strategies are not constrained by force data on disease related radiological models and often have higher expressive precision than progressively standard quantitative examination in perspective on direct volume or thickness measures. In any case the trading of fundamentally wiped out patients requires incredible coordination to give the definite gadgets and the most reasonable treatment for their conditions. Thusly it is fundamental to portray the contamination on schedule and thusly offering prescriptions to the patients to peril avoidance. Every so often same properties with comparable symptoms of ailments required assorted medications. Thusly precise decisions and portrayal of data is required.

The SVM endeavors to decide a tradeoff between limiting the preparation set mistake and boosting the edge so as to accomplish the best speculation capacity and stay impervious to over fitting. Because of its favorable nature, SVM has been connected to a wide scope of There precise choices and characterization of information is required. According to the diagram drove in 2017, in just us there are 252,710 occurrences of chest malady. So the amount of chest development all around the world will be a regard amazingly tremendous. In US the passing rate of women as a result of chest danger is higher diverged from other malady arrangements. After skin harm chest illness is most generally found among women. In India it is found that chest tumor is as of now by and large found in progressively young age bundles as well. It is the most outstanding kind of tumor in Indian urban domains and second customary in provincial zones.

\section{CASE STUDY DESIGN}

The main intension of the study is to classify the medical data with high accuracy. The primary objective of this Case Study work is to develop computer aided systems to assist clinicians in decision making. To achieve the objective of study, we present a conceptual framework for designing case study. The data we study is collected from patients to breast cancer disease. The overall process of Case Study can be divided in to four stages,

Stage 1: Preprocessing

Stage 2: Optimal Feature selection using KHA

Stage 3: Classification using HAKNN

Stage 4: Case study Comparison

The detailed process of the A Case Study approach using Hybrid Adaboost KNN is shown in Figure 2.

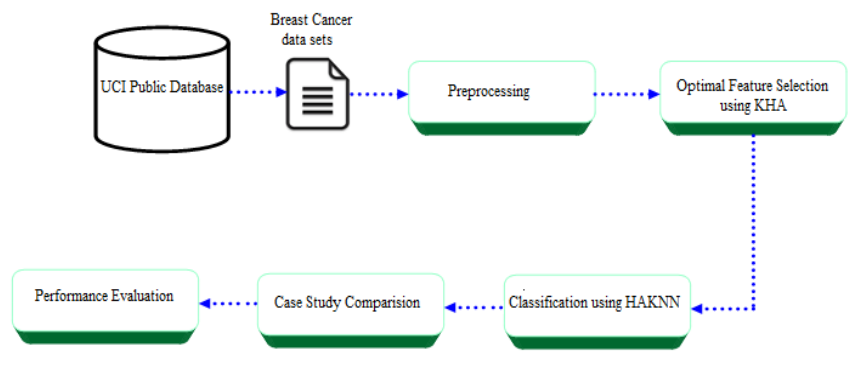

Figure 2. A case study approach using hybrid adaboost KNN

\section{Preprocessing}

Preprocessing is most important step in data mining, which collect the pre-processing will be applied to extract useful data and to convert suitable sample from raw medical datasets. In preprocessing, the non-numerical data and missing values are removed from the input dataset and obtained the numerical dataset. The preprocessed output is fed to the further process.

\section{OPTIMAL FEATURE SELECTION USING KRILL HERD ALGORITHM (KHA)}

After preprocessing optimal features are selected by using 
krill Herd Algorithm (KHA). KHA is a streamlining technique to tackling enhancement issues that depends on krill herd swarms of organic and ecological forms.

\subsection{Krill Herd Algorithm (KHA), initialization}

The main parameters of the Krill Herd Algorithm(KHA) are the total evolution number, the population size, $D^{\max }$ (Maximum induced motion), $S_{f}$ (Sensing factor), $r_{t}$ ( Random number ) and $R_{D}^{\max }$ ( Maximum diffusion speed). In this technique, Krill Herd represents the features value.

\subsection{Fitness calculation}

Evaluate the fitness utility depends on the maximum accuracy and moreover select the finest result.

Fitness $=$ Max(accuracy)

three movement of each individual krill are,

(a) krill individuals Development,

(b) Foraging action,

(c) Random dispersion.

\subsection{Development initiated by other Krill individuals}

Speed of every krill individual is impacted by the development of the other Krill's to keep up a high thickness. Three impacts namely neighborhood impact (x), target impact (y) and repulsive impact ( $\mathrm{z}$ ) are used to evaluate the direction of motion induced $\left(\xi_{m}\right)$. Krill individual motion may be formulated as

$$
D_{m}^{\text {new }}=\xi_{m} D^{\max }+\chi_{b} D_{m}^{\text {old }}
$$

The sensing distance $S_{d}$ between the individual krills and the neighbors formulated by,

$$
S_{d}=\frac{1}{5 N} \sum_{n=1}^{N-1}\left|F_{m}-F_{n}\right|
$$

\subsection{Foraging action}

The foraging velocity of $m^{\text {th }}$ krill individual can be expressed by

$$
F_{F m}^{n e w}=S_{f} \varsigma_{m}+\chi_{x} F_{F m}^{o l d}
$$

\subsection{Random dispersion}

To enhance the population diversity random diffusion process is mainly considered and it is expressed by

$$
R_{D m}^{\text {new }}=\beta \times R_{D}^{\max }
$$

\subsection{Updating the krill position}

In updating the position, the individual krill changes its present positions also, moves to better positions in light of induction movement, foraging movement and random dispersion movement. In this three movements, the upgraded position of the $m^{\text {th }} \mathrm{krill}$ individuals during the interval of $\mathrm{t}$ and $\Delta t$ might be communicated by
$P_{m}(t+\Delta t)=P_{m}(t)+\Delta t \frac{d P_{m}}{d t}$

Based on the above procedure, we select the optimal features and then the selected features are fed to the classification process.

\subsection{Classification using Hybrid Adaboost KNN algorithm}

Finally, the optimal features are furnished to Hybrid Adaboost KNN classifier for the purpose of classification.

\subsection{Hybrid Adaboost KNN algorithm}

Hybrid Adaboost technique combine multiple "weak classifiers" into a single "strong classifier". Each weak classifier should be trained on a random subset of the total training set. Adaboost assigns a "weight" to each training sample, which determines the probability that each sample should appear in the training set. After training a classifier, Adaboost increases the weight of the classifier. All the learners are simple and weak and must have error less than 0.5 . Otherwise, the process is stopped since its continuation makes the learning become difficult for the next classifier. Also, the initial probability of selecting sample is considered to be uniform. In fact, the weight of sample shows the importance of the sample. The final hypothesis is obtained through weighted voting of $T$ number of weak hypotheses. The steps involved in this algorithm are shown below.

Step 1: Initialization of weight $W$

Step 2: In the case, $t \leq$ Tanderr $^{t}<0.5$, then normalize weight $W^{t}$, so that, $\sum_{t=1}^{N} W_{i}^{t}=1$

Step 3: Call KNN, providing with the weight $W^{t}$, get hypothesis $h^{t}: X->\{-1,1\}$

\subsection{Krill Nearest Neighbor (KNN) classifier}

The KNN calculation is a strategy which is utilized for characterization of any items or different components dependent on the nearest preparing information which are accessible in the element space. The qualities closest to the Krill esteem will be picked for the characterization results. When the grouping of closest neighbor is done, convert it into vector esteems with fixed length by using the Euclidean separation work in $\mathrm{KNN}$ which is given in beneath articulation,

$E_{D}(x, y)=\left(\sum_{k=1}^{N}\left(x_{k}-y_{k}\right)^{2}\right)^{\frac{1}{2}}$

where $\mathrm{x}, \mathrm{y}$ are feature values;

The premise of the KNN classifier is the little neighborhood in the comparable highlights. These procedures will give better exactness in arranging the outcomes.

Step 4: Compute $\operatorname{err}^{t}=\sum_{i=1}^{N} W_{i}^{t} e_{i}^{t}$,

Where $e_{i}^{t}=1$, if $h^{t}\left(x_{i}\right) \neq v_{i}$, and 0 otherwise

Step 5: Set $\alpha^{t}=0.5 \log \left[\left(1-\right.\right.$ err $\left.^{t}\right) /$ err $\left.^{t}\right]$

Step 6: Update the weights to be as follows, $W_{i}^{t+1}=W_{i}^{t} \exp \left(2 \alpha^{t} e^{t}\right)$

Step 7: Put $T=t+1$ and the process repeats until err $=0$.

After every classifier is prepared, the classifier's weight is determined dependent on its exactness. Increasingly precise 
classifiers are given more weight. At long last we characterize the medicinal information with high precision esteem.

\section{RESULTS AND DISCUSSION}

The Case Study of Medical classification is implemented using Python software and the experiment is done using i5 processor with 3GB RAM.

\subsection{Dataset description}

\subsubsection{Mammographic mass data set}

These datasets are taken from the UCI machine learning repository. The database comprises of around 2,620 cases.

For each case, dual images of every breast, inter related patient information, like age, period of the tumor, subtlety rating for varieties from the standard, American College of Radiology (ACR) breast thickness rating are considered. The Mammograms are digitized by various scanners depending upon the wellspring of the data.

\subsection{Evaluation metric}

The evaluation metrics used here contains True Positive, True Negative, False Positive and False Negative, Sensitivity, Specificity and Accuracy.

$$
\begin{aligned}
\text { Sensitivity } & =\frac{\mathrm{T}(\mathrm{P})}{\mathrm{T}(\mathrm{P})+\mathrm{F}(\mathrm{N})} \\
\text { Specificity } & =\frac{\mathrm{T}(\mathrm{N})}{\mathrm{F}(\mathrm{P})+\mathrm{T}(\mathrm{N})} \\
\text { Accuracy } & =\frac{\mathrm{T}(\mathrm{P})+\mathrm{T}(\mathrm{N})}{\mathrm{T}(\mathrm{P})+\mathrm{F}(\mathrm{N})+\mathrm{F}(\mathrm{P})+\mathrm{T}(\mathrm{N})}
\end{aligned}
$$

\subsection{Case study comparative analysis}

We can build up that our Case Study achieves great exactness for the medical data classification. Hybrid Adaboost Algorithm together with $\mathrm{KNN}$ algorithm is utilized for medical data classification in our investigation strategy.

And furthermore we can set up this forecast precision result by contrasting different classifiers. Case Study classifier is contrasted with the Hybrid Adboost KNN along with Krill Herd Algorithm (KHA), existing Ant Colony Optimization (AOC) and Support Vector Mechanism (SVM) classifiers. The Comparison results are introduced in the Table 1.

Table 1. Case study comparative analysis of the proposed and existing method

\begin{tabular}{|c|c|c|c|c|c|c|}
\hline \multirow{2}{*}{ Datasets } & \multicolumn{3}{|c|}{$\begin{array}{c}\text { Proposed Method } \\
\text { (KHA+HAKNN) }\end{array}$} & \multicolumn{3}{c|}{$\begin{array}{c}\text { Existing Method } \\
\text { (AOC+SVM) }\end{array}$} \\
\cline { 2 - 7 } & Accuracy & Sensitivity & Specificity & Accuracy & Sensitivity & Specificity \\
\hline $\begin{array}{c}\text { Mammographic } \\
\text { Mass data }\end{array}$ & $95.86 \%$ & $98.5 \%$ & $96.5 \%$ & $87.1 \%$ & $89.3 \%$ & $94.45 \%$ \\
\hline
\end{tabular}

\section{Accuracy:}

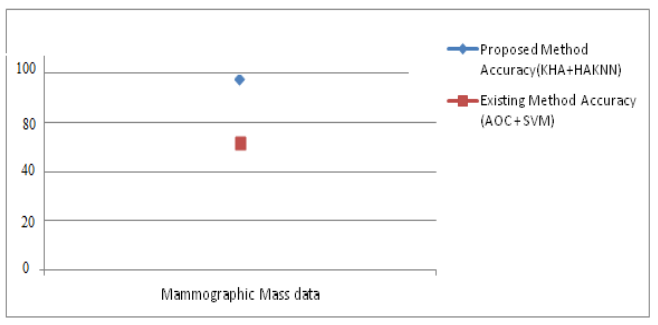

Figure 3. The comparison outcomes of the Accuracy measure

\section{Sensitivity:}

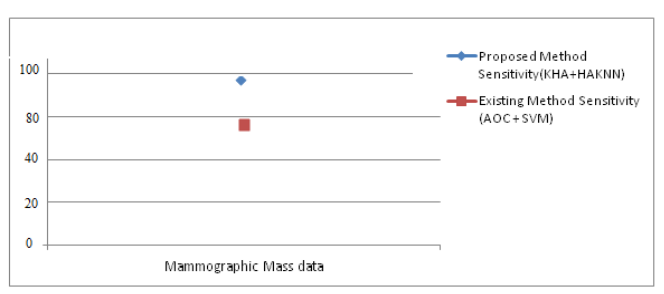

Figure 4. The comparison outcomes of the Sensitivity measure

\section{Specificity:}

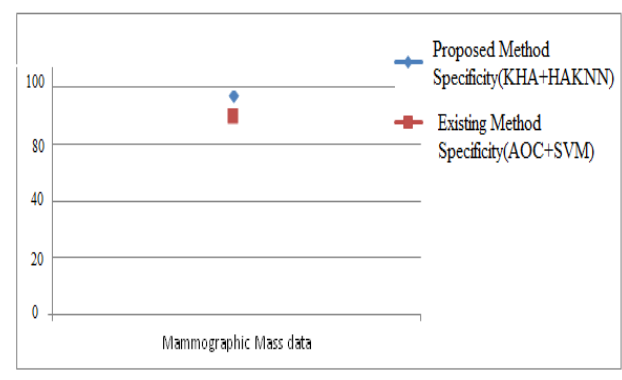

Figure 5. The comparison outcomes of the Specificity measure

\subsection{Performance analysis}

The results of case study assist in analyzing the effectiveness of the prediction method. The results of the breast cancer datasets are provided in below table 2

Table 2. Performance analysis of the proposed method

\begin{tabular}{|c|c|c|c|}
\hline Dataset & Accuracy & Sensitivity & Specificity \\
\hline $\begin{array}{c}\text { Mammographic } \\
\text { Mass data }\end{array}$ & $95.86 \%$ & $98.5 \%$ & $96.5 \%$ \\
\hline
\end{tabular}

From Table 1, it is clear that the accuracy value for mammographic mass data obtained using the proposed method is $95.86 \%$, similarly the sensitivity and specificity value obtained is $98.5 \%$ and $97.6 \%$ respectively. 


\section{CONCLUSION}

Breast cancer is danger disease among ladies around the world. Case Study investigation technique of Breast cancer data classification can be done with help of optimal feature selection. Krill Herd Algorithm (KHA) is used to selecting optimal features. The optimal features are classified with Hybrid Adaboost KNN classification algorithm. Experimentation data sets are collected from UCI machine learning public database. Case Study performance evaluated by utilizing accuracy, sensitivity and specificity. The Study investigation Hybrid Adaboost KNN technique achieve the maximum accuracy, sensitivity and specificity were $95.86 \%$, $98.5 \%$ and $96.5 \%$. Case Study investigation implemented using python software.

\section{REFERENCES}

[1] Siegel R, Ma J, Zou Z, Jemal A. (2014). Cancer statistics. CA: A Cancer Journal for Clinicians 64(1): 9-29.

[2] Bhardwaj A, Tiwari A. (2015). Breast cancer diagnosis using Geneticallyoptimized neural network model. Expert Syst. Appl. 42(10): 4611-4620. http://dx.doi.org/10.1016/j.eswa.2015.01.065

[3] Jemal A, Bray F, Center MM, Ferlay J, Ward E, Forman D. (2011). Global cancer statistics. CA, A Cancer J. Clinicians 61(2): 69-90.

[4] Youlden DR, Cramb SM, Dunn NA, Müller JM, Pyke CM, Baade PD. (2012). The descriptive epidemiology of female breast cancer: An international comparison of screening, incidence, survival and mortality. Cancer Epidemiol 36(3): 237-248. http://dx.doi.org/10.1016/j.canep.2012.02.007

[5] Saghir NSE, Khalil MK, Eid T, Kinge ARE, Charafeddine M, Geara F, Seoud M, Shamseddine AI. (2007). Trends in epidemiology and management of breast cancer in developing Arab countries: A literature and registry analysis. International Journal of Surgery 5(4): 225-233. https://doi.org/10.1016/j.ijsu.2006.06.015

[6] Ravichandran K, Al-Zahrani AS. (2009). Association of reproductive factors with the incidence of breast cancer in Gulf cooperation council countries. East Mediterr. Health $\quad$ J. $\quad 15(3)$ : $\quad 612-621$. http://dx.doi.org/10.1002/dev.20373

[7] Thompson D, Easton D. (2004). The genetic epidemiology of breast cancer genes. J. Mammary Gland. Biol. Neoplasia 9(3): 221-236. http://dx.doi.org/10.1023/B:JOMG.0000048770.90334. $3 b$

[8] Bray F, McCarron P, Parkin DM. (2004). The changing global patterns of female breast cancer incidence and mortality. Breast Cancer Res 6(6): 229-239. https://doi.org/10.1186/bcr932

[9] Parkin DM, Bray F, Ferlay J, Pisani P. (2005). Global cancer statistics, 2002. CA, Cancer J. Clinicians 55(2): 74-108.

[10] McPherson K, Steel CM, Dixon KM. (2000). Breast cancer_Epidemiology, risk factors, and genetics. BMJ 321(7261): 624-628.

[11] Perera NM, Gui GP. (2003). Multi-ethnic differences in breast cancer: Current concepts and future directions. Int. J. Cancer 106(4): 463-467. http://dx.doi.org/10.1002/ijc.11237

[12] Ezzat AA, Ibrahim EM, Raja MA, Al-Sobhi S, Rostom A, Stuart RK. (1999). Locally advanced breast cancer in Saudi Arabia: High frequency of stage III in a young population. Med. Oncol 16(2): 95-103. http://dx.doi.org/10.1007/bf02785842

[13] Ibrahim EM, Ezzat AA, Rahal MM, Raja MM, Ajarim DS. (2005). Adjuvant chemotherapy in 780 patients with early breast cancer: 10-yeardata from Saudi Arabia. Med. Oncol. 22(4): 343-352. http://dx.doi.org/10.1385/mo:22:4:343

[14] Elkum N, Dermime S, Ajarim, D, Alzahrani A, Alsayed A, Tulbah A, Al Malik O, Alshabanah M, Ezzat A, Al Tweigeri T. (2007). Being 40 or younger is an independent risk factor for relapse in operable breast cancer patients: The Saudi Arabia experience. BMC Cancer 7: 222. http://dx.doi.org/10.1186/1471-2407-7222

[15] Najjar H, Easson A. (2010). Age at diagnosis of breast cancer in Arab nations. Int. J. Surg 8(6): 448-452. http://dx.doi.org/10.1016/j.ijsu.2010.05.012

[16] Farr A, Wuerstlein R, Heiduschka A, Singer CF, Harbeck N. (2013). Modern risk assessment for individualizing treatment concepts in early-stage breast cancer. Rev. Obstetrics Gynecol 6(3-4): 165-173.

[17] Fayyad U, Piatetsky-Shapiro G, Smyth P. (1996). From data mining to knowledge discovery in databases. AI Mag 17(3): 37. 